\title{
SUBMILLIMETER OBSERVATIONS OF RADIO-QUIET QUASARS AND A LUMINOUS SEYFERT 1 GALAXY INCLUDING THE FIRST DETECTIONS
}

\author{
RICHARD BARVAINIS \\ MIT/Haystack Observatory, ${ }^{1}$ Westford, MA 01886 \\ ROBERT ANTONUCCI \\ Physics Department, University of California, Santa Barbara, CA 93106 \\ AND \\ Paul Coleman \\ Sterrewacht Leiden, Postbus 9513, 2300 RA Leiden, The Netherlands \\ Received 1992 July 21; accepted 1992 August 19
}

\begin{abstract}
We report submillimeter continuum observations of six radio-quiet active galactic nuclei selected for high $I R A S$ flux, in order to test models for the infrared continuum by constraining the far-infrared/submillimeter cutoff slope. Slopes greater than the self-absorbed synchrotron cutoff $\alpha_{\text {cutoff }}=2.5\left(f_{v} \propto v^{\alpha}\right)$ argue against the synchrotron model and lend support to the dust model. (The synchrotron cutoff slope can be slightly sharper than 2.5 if concave electron energy distributions and extreme homogeneity are assumed.) We detected the luminous Seyfert 1 galaxy PG $1434+590$ at 438 and $345 \mu \mathrm{m}$; combining these detections with the IRAS 100 $\mu \mathrm{m}$ flux gives a slope of $2.53 \pm 0.17$. Using our observed submillimeter upper limits for the radio-quiet quasars, we were able to constrain the cutoff slopes such that $\alpha_{\text {cutoff }}>2.50$ in two cases (data for three others do not provide significant slope constraints). We conclude that the dust model is favored, but that synchrotron emission cannot yet be ruled out conclusively.

In a related program we also observed and detected the $z=2.546$ gravitationally lensed quasar $\mathrm{H} 1413+117$, the "cloverleaf," at 345,438 , and $761 \mu \mathrm{m}$. The slope between 438 and $761 \mu \mathrm{m}$ (observed; $124-$ $215 \mu \mathrm{m}$ rest frame) in this object is found to be $2.95 \pm 0.69$. We suggest that in $\mathrm{H} 1413+117$ we may be seeing a highly luminous but otherwise rather normal far-infrared peak shifted well into the submillimeter region.

Subject headings: galaxies: Seyfert - quasars: general — radiation mechanisms: thermal
\end{abstract}

\section{INTRODUCTION}

Two models are currently popular for explaining the origin of the infrared emission from radio-quiet and non-blazar radio-loud quasars and active galactic nuclei (AGNs). These are the nonthermal (synchrotron) and thermal (dust) models. While there are several kinds of observations which might help distinguish between these models (see Barvainis 1992 for a review), one of the cleanest tests involves the far-infrared/ submillimeter cutoff slope. This test is based on the theoretical expectation that the shape of the spectrum at wavelengths longward of the turnover $(\sim 100 \mu \mathrm{m})$ will be different for the synchrotron and dust models. The former has a maximum cutoff slope of $\alpha=2.5\left(f_{v} \propto v^{\alpha}\right)$ for self-absorbed emission from electrons with a simple power-law energy distribution, or somewhat larger $(\alpha \lesssim 3)$ if a thermal electron pool or dual power-law energy distribution is invoked (de Kool \& Begelman 1989; Schlickeiser, Biermann, \& Crusius-Watzel 1991). In either case the maximum synchrotron slope is attained only for a completely homogeneous source (e.g., Sanders et al. 1989). The asymptotic dust slope, however, should be $\gtrsim 3$ since the optically thin spectrum derives from the Rayleigh-Jeans law times the dust emissivity law, the latter having the form $Q_{v} \propto v^{1-2}$ (e.g., Draine \& Lee 1984). (Slopes measured near the turnover can, of course, be shallower.) Observationally all galaxies with far-infrared emission from dust show these steep slopes.

\footnotetext{
${ }^{1}$ Radio Astronomy at the Haystack Observatory of the Northeast Radio Observatory Corporation (NEROC) is supported by a grant from the National Science Foundation.
}

The cutoff slope test was first exercised in a meaningful way by Edelson et al. (1988) and Chini, Kreysa, \& Beirmann (1989), who made observations at 438 and $1300 \mu \mathrm{m}$, respectively, for comparison with $I R A S 100 \mu \mathrm{m}$ detections. Edelson et al. derived a cutoff slope of greater than 3.06 for the intermediate luminosity Seyfert 1 galaxy NGC 4151, while Chini et al. observed a number of quasars and found lower limits to cutoff slopes as large as 2.64 . In this paper we describe a program to try to determine turnover shapes and cutoff slopes for several quasars and a luminous Seyfert galaxy via observations in the submillimeter region, at wavelengths of 345-1300 $\mu \mathrm{m}$.

\section{OBSERVATIONS}

We used the UKT14 bolometer (Duncan et al. 1990) on the $15 \mathrm{~m}$ James Clerke Maxwell Telescope (JCMT) located on Mauna Kea, Hawaii, during 1991 February 8-11 and 1992 March 20-22 for the observations. During the 1991 run a number of instrumental and weather problems prevented us from obtaining useful results except for one source, PG $1434+590$ at $438 \mu \mathrm{m}$. For those observations, absolute flux was calibrated using Uranus, and atmospheric attenuation was measured by following Uranus as it set over the range $\approx 3-4$ air masses. The zenith opacity at $438 \mu \mathrm{m}$ was in the range $0.6-1.2$ nepers. Pointing was checked every $2-3 \mathrm{hr}$ on $3 \mathrm{C} 345$. The observations with UKT14 were performed using a chopping secondary mirror, with a chop frequency of $7.8 \mathrm{~Hz}$.

The 1992 observations, by contrast, were virtually troublefree, and the weather was excellent for most of the run. The 
chop frequency was the same as for the 1991 observations. Absolute flux calibrations were derived primarily from observations of Mars. The atmospheric attenuation during the 1992 Iun was derived from continuous opacity measurements made at $1300 \mu \mathrm{m}$ by a tipping radiometer operated by the Caltech Submillimeter Observatory. Zenith opacities at the observed wavelengths were computed from the $1300 \mu \mathrm{m}$ measurements using known scaling factors. The nighttime zenith opacities at $1300 \mu \mathrm{m}$ were $0.03-0.04$ nepers, with afternoon values being typically a factor of 2-3 higher. The scale factors relating $\tau_{1300}$ to $\tau$ at the observed wavelengths were adopted to be 22.0 at 345 and $438 \mu \mathrm{m}$ (e.g., $\left.\tau_{438}=22.0 \tau_{1300}\right), 6.0$ at $761 \mu \mathrm{m}$, and 1.5 at $1100 \mu \mathrm{m}$ (B. Duncan 1992, private communication). The observed wavelengths quoted here refer to the centers of the UKT14 filters, defined as the average of the wavelengths at which the filter response is $50 \%$ of its maximum. The actual effective wavelengths of observation depend on wavelengthdependent variables such as atmospheric transmission, antenna efficiency, and source spectral index. We have not attempted to calculate these effective wavelengths, but given the other uncertainties in the fluxes, such as statistical and calibration errors, errors in spectral shape resulting from uncertainties in the effective wavelengths should be relatively small.

\section{RESULTS}

As mentioned above, in 1991 we were able to observe only one source at one wavelength, that being the luminous Seyfert 1 galaxy PG $1434+590$ at $438 \mu \mathrm{m}$. In 1992 we observed six radio-quiet $A G N s$ (including $P G 1434+590$ ) selected for their relatively high $100 \mu \mathrm{m} I R A S$ fluxes, and luminosities close to or above the cutoff defined for quasars by Schmidt \& Green (1983; $M_{V}=-23$ for $H_{0}=50 \mathrm{~km} \mathrm{~s} \mathrm{Mpc}^{-1}$, or $M_{V}=$ -22.1 for $H_{0}=75 \mathrm{~km} \mathrm{~s}^{-1} \mathrm{Mpc}^{-1}$, the value assumed in this Letter). The $100 \mu \mathrm{m}$ selection criterion allows for better slope constraints when combining IR AS and submillimeter data, but may bias the sample toward quasars which are unusually dusty. As part of a related program (Coleman 1992), we also observed several broad-absorption-line quasars (BALQs), which, like all known members of their class, are also radio quiet. The thrust of that program was to determine if BALQs are universally radio quiet at least in part because of absorption of any radio emission by an optically thick free-free screen. Such a screen should become transparent in the millimeter/ submillimeter region, allowing the radio source to be seen. We report here submillimeter detections of one of the BALQs, because in one interpretation this result is closely related to the original program to study radio-quiet AGN turnovers (see $\S 4.2$ below). The sources observed are listed in Table 1 , which includes their redshifts and apparent and absolute visual magnitudes.

The measured flux densities and upper limits are given in Table 2. Quoted uncertainties for the detections are statistical and do not include calibration errors. These errors, caused by variations in the telescope efficiency, the pointing, and the atmospheric attenuation, become worse toward shorter wavelengths. We estimate the calibration errors to be in the range $10 \%-20 \%$ at 761 and $1300 \mu \mathrm{m}$, and $20 \%-30 \%$ at 345 and 438 $\mu \mathrm{m}$. For the upper limits, we quote 2.5 times the statistical uncertainty, plus the calibration uncertainty added in quadrature. Also listed in Table 2 are the $100 \mu \mathrm{m}$ flux densities and derived cutoff slope constraints. The errors on the slopes include both statistical and calibration uncertainties.
TABLE 1

SOURCE LIST

\begin{tabular}{cllccc}
\hline \hline $\begin{array}{c}\text { Coordinate } \\
\text { Name }\end{array}$ & \multicolumn{1}{c}{$\begin{array}{c}\text { Other } \\
\text { Name }\end{array}$} & Type $^{\mathrm{a}}$ & \multicolumn{1}{c}{$z$} & $m_{\boldsymbol{v}}{ }^{\mathrm{b}}$ & $M_{\boldsymbol{v}}{ }^{\mathrm{b}}$ \\
\hline $0157+001 \ldots \ldots \ldots$ & Mrk 1014 & RQQ & 0.163 & 15.69 & -23.3 \\
$0710+457 \ldots \ldots \ldots$ & Mrk 376 & RQQ & 0.056 & 14.62 & -22.1 \\
$0759+651 \ldots \ldots \ldots$ & IR $0759+6508$ & RQQ & 0.148 & 15.5 & -23.3 \\
$1402+437 \ldots \ldots \ldots$ & IR $1402+4341$ & RQQ & 0.320 & 16.5 & -24.2 \\
$1413+117 \ldots \ldots \ldots$ & H1413+117 & BAL & 2.546 & 17.0 & -28.9 \\
$1434+590 \ldots \ldots \ldots$ & PG $1434+590$ & SY1 & 0.033 & 13.9 & -21.7 \\
$1821+643 \ldots \ldots \ldots$ & E1821+643 & RQQ & 0.297 & 14.1 & -26.3 \\
\hline
\end{tabular}

${ }^{a}$ RQQ or SY1 indicate radio-quiet quasar or Seyfert 1 galaxy selected for high IRAS flux; BAL indicates source selected for broad absorption lines.

${ }^{b}$ From Veron-Cetty \& Veron 1989. $M_{V}$ has been adjusted for $H_{0}=75 \mathrm{~km}$ $\mathrm{s}^{-1} \mathrm{Mpc}^{-1}$.

Two sources were detected in the submillimeter. The luminous Seyfert 1 galaxy PG $1434+590$ was detected at $438 \mu \mathrm{m}$ in both the 1991 and 1992 observations. The measured values were $102 \pm 25 \mathrm{mJy}(1991)$ and $60 \pm 11 \mathrm{mJy}$ (1992), which appear to be just consistent with each other given the statistical and calibration uncertainties (the latter not included in the quoted errors). The weighted average for the two years is $67 \pm 10 \mathrm{mJy}$. This source was also likely detected at $345 \mu \mathrm{m}$, at the $2.9 \sigma$ level. The submillimeter, infrared, and optical spectral energy distribution (SED) of PG $1434+590$ is shown in Figure 1.

The other source detected was the BALQ H1413+117. This object has a much higher redshift $(z=2.546)$ than any of the other sources, and it is also gravitationally lensed (Magain et al. 1988). Clear detections were obtained at 761, 438, and 345

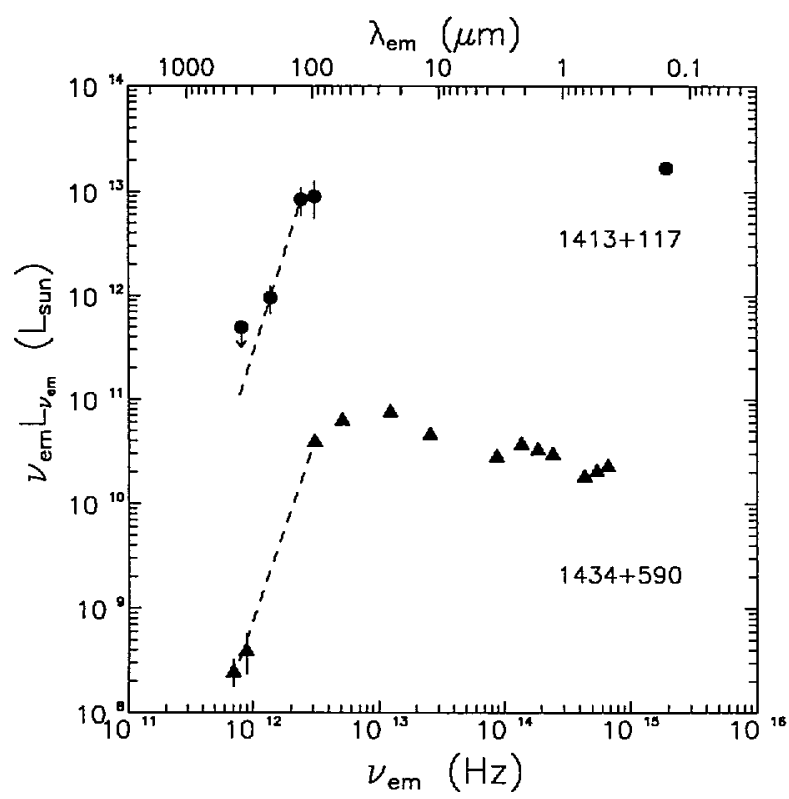

F1G. 1.-Rest-frame spectral energy distributions for the far-infrared-selected AGN PG $1434+590$, and the broad-absorption-line quasar H1413+117. Note that the far-infrared/submillimeter cutoff occurs at the same place in both objects. Optical datum for $\mathrm{H} 1413+117$ is from Veron-Cetty \& Veron (1989). Optical data for $1434+590$ are from Edelson \& Malkan (1986), near-infrared fluxes are from Rudy et al. (1982), and IRAS fluxes are from Barvainis \& Antonucci (1989). Error bars on the submillimeter points reflect both statistical and calibration uncertainties. 
TABLE 2

MEASUREMENTS, $100 \mu \mathrm{m}$ IRAS Fluxes, AND Slope CONSTRAints

\begin{tabular}{ccccccc}
\hline \hline \multirow{2}{*}{ OВJECT } & \multirow{7}{c}{$f_{v}(\mathrm{mJy})^{\mathrm{a}}$} \\
\cline { 2 - 6 } & $1300 \mu \mathrm{m}$ & $761 \mu \mathrm{m}$ & $438 \mu \mathrm{m}$ & $345 \mu \mathrm{m}$ & $100 \mu \mathrm{m}$ & $\alpha_{\text {cutoff }}{ }^{\mathrm{b}}$ \\
\hline $0157+001 \ldots \ldots \ldots$ & $\ldots$ & $<41$ & $\ldots$ & $\ldots$ & $2322 \pm 130$ & $>1.99$ \\
$0710+457 \ldots \ldots \ldots$ & $\ldots$ & $<16$ & $\ldots$ & $\ldots$ & $1439 \pm 107$ & $>2.22$ \\
$0759+651 \ldots \ldots \ldots$ & $\ldots$ & $<19$ & $<42$ & $\ldots$ & 1810 & $>2.55$ \\
$1402+437 \ldots \ldots \ldots$ & $\ldots$ & $\ldots$ & $<106$ & $\ldots$ & 1150 & $>1.61$ \\
$1413+117 \ldots \ldots \ldots$ & $<35$ & $44 \pm 8$ & $224 \pm 38$ & $189 \pm 56$ & $\ldots$ & $2.95 \pm 0.69$ \\
$1434+590 \ldots \ldots \ldots$ & $\ldots$ & $\ldots$ & $67 \pm 10^{\mathrm{c}}$ & $84 \pm 29$ & $2443 \pm 82$ & $2.53 \pm 0.17$ \\
$1821+643 \ldots \ldots \ldots$ & $\ldots$ & $\ldots$ & $<45$ & $\ldots$ & $2164 \pm 82$ & $>2.62$ \\
\hline
\end{tabular}

a Upper limits are $2.5 \sigma$ plus calibration uncertainties added in quadrature. Quoted errors for the detections are statistical and do not include calibration uncertainties. IRAS $100 \mu \mathrm{m}$ data from Neugebauer et al. $1986(0157+001,0710+457$, and $1821+643)$; Low et al. $1988(0759+651$ and $1402+434$; no errors given); and Barvainis \& Antonucci $1989(1434+590)$.

${ }^{b}$ Lower limit to cutoff slope between submillimeter and $100 \mu \mathrm{m}$ points. For $0759+651$, the slope is measured between 100 and $438 \mu \mathrm{m}$. For $1434+590$, the quoted slope is for the best-fit power between the 100,345 , and $438 \mu \mathrm{m}$ points. For $1413+117$ the slope is between the $438 \mu \mathrm{m}$ and $761 \mu \mathrm{m}$ points. The errors on the slopes include both statistical and calibration uncertainties.

c Weighted average of measurements in 1991 and 1992.

$\mu \mathrm{m}$, with a weak upper limit at $1300 \mu \mathrm{m}$. The SED is shown in Figure 1.

\section{DISCUSSION}

\subsection{The IRAS-Selected AGNs}

Two of the nondetected sources, IR $0759+6508$ and E1821 + 643, have interesting cutoff slope limits, in that they essentially rule out the traditional single power-law selfabsorbed synchrotron (SAS) model, which has a maximum cutoff slope of 2.50. (This has been done previously for several other quasars by Chini et al. 1989 using $1300 \mu \mathrm{m}$ observations). However, it appears possible to obtain slopes greater than 2.50 in the SAS model. To this end de Kool \& Begelman (1989) postulated a thermal electron background in a synchrotron source, while Schlickeiser et al. (1991) invoked a double-powerlaw electron energy distribution. In the latter model, SAS slopes $\gtrsim 3$ require differences in electron energy slopes $\gtrsim 5$. Even for $\alpha_{\text {SAS }}=2.62$, an example used by Schlickeiser et al. and equal to our lower limit for E1821 +643, the electron energy slope difference must be greater than 2.4. Since even minor source inhomogeneities will quickly reduce the SAS cutoff slope (Marscher 1979; Sanders et al. 1989), we consider the results presented here, and those of Chini et al. (1989) and Edelson et al. (1988), to be unfavorable to the synchrotron model, although not yet able to rule it out completely.

The only one of the IRAS-selected AGNs detected in the submillimeter was PG $1434+590$. This was also the only Seyfert galaxy observed, but it is quite luminous for its class (being only $0.4 \mathrm{mag}$ fainter than the generally accepted cutoff for quasars). Like most quasars, its infrared spectrum does not look much like those of IRAS galaxies, whose steep 25-60 $\mu \mathrm{m}$ slopes are characteristic of "thermal" sources (see Fig. 5 of Barvainis \& Antonucci 1989). However, this does not necessarily imply that the infrared is nonthermal radiation. It has been shown that the sort of mildly convex far-infrared SED demonstrated by $P G 1434+590$ is naturally explained by multitemperature dust at a range of distances from a central optical/UV heating source (Barvainis 1987, 1990; Sanders et al. 1989). The best-fit slope of $2.53 \pm 0.17$ for the 100,345 , and 438 $\mu \mathrm{m}$ data for PG $1434+590$ again pushes hard on the synchrotron theory and is in fact a lower limit to the true cutoff slope since the spectrum has not yet turned over completely by 100 $\mu \mathrm{m}$. It is quite reasonable, however, for the dust model, since, as for inhomogeneities in a synchrotron source, the dust slope will be softened somewhat in the turnover region by multiple grain temperatures. More stringent slope constraints can potentially be obtained by combining our detections with other submillimeter measurements in the future. We plan to pursue this topic using the Kuiper Airborne Observatory at $155 \mu \mathrm{m}$ in the near term, and with SCUBA, a sensitive submillimeter camera being built for use on the JCMT and proposed for operation in the 1-2 yr time frame. We are also using the IRAM $30 \mathrm{~m}$ telescope for photometry at $1300 \mu \mathrm{m}$.

\subsection{The BALQ H1413+117}

$\mathrm{H} 1413+117$ is the famous "cloverleaf" quasar, which is unique in being the only known gravitationally lensed broadabsorption-line object. Coleman (1992) discusses the submillimeter detection of $\mathrm{H} 1413+117$ in the context of the free-free absorption model, but here we emphasize another interpretation. Examination of the spectrum in Figure 1 shows that the submillimeter continuum appears to turn over somewhere between 345 and $761 \mu \mathrm{m}$ in the observed frame, or between 97 and $215 \mu \mathrm{m}$ in the rest frame. This is precisely the region where the far-infrared/submillimeter turnover occurs for nearby objects such as $P G 1434+590$ and the other IRAS AGNs discussed above. Therefore, we propose that in $\mathrm{H} 1413+117$ we are seeing the usual sort of far-infrared peak found in $I R A S$-selected quasars, but shifted well into the submillimeter region. As a rough consistency check one can ask, is the ratio of $100 \mu \mathrm{m}$ to $V$ band flux compatible with that found in relatively nearby objects? Taking the seven objects in Table 2 with $100 \mu \mathrm{m}$ detections, we find a range for this ratio of $168<$ $F_{100} / F_{V}<1292$, with a mean value $\left\langle F_{100} / F_{V}\right\rangle=617$. Taking the $345 \mu \mathrm{m}$ value of $189 \mathrm{mJy}$ for the $100 \mu \mathrm{m}$ (actually $97 \mu \mathrm{m}$ rest frame) flux, and deredshifting the observed $V$ flux $(=0.56$ $\mathrm{mJy}$ ) assuming an optical/UV power law with slope -0.5 , we find $F_{100} / F_{V}=630$ for $\mathrm{H} 1413+117$. The closeness of this ratio in $\mathrm{H} 1413+117$ to the mean value found for the other (farinfrared selected) objects is almost certainly fortuitous, but this exercise at least demonstrates consistency of the spectral shape within our interpretation. 
If we are indeed observing the far-infrared turnover (as opposed to free-free absorption) in $\mathrm{H} 1413+117$, then the cutoff slope of $2.95 \pm 0.69$ between rest wavelengths 124 and $215 \mu \mathrm{m}$ (438 and $761 \mu \mathrm{m}$ observed) is the steepest slope constraint we are currently able to set using our data. Again this is probably a lower limit, since there is an indication that the turnover may occur longward of $124 \mu \mathrm{m}$. The submillimeter properties of $\mathrm{H} 1413+117$ are quite similar to those of the extreme high-redshift IRAS galaxy IR $10214+4724(z=2.286$; compare with $z=2.546$ for $\mathrm{H} 1413+117$ ). For this object the 800 and $450 \mu \mathrm{m}$ fluxes are $30 \pm 7 \mathrm{mJy}$ and $365 \pm 100 \mathrm{mJy}$, yielding a slope of $4.3 \pm 0.5$ (Clements et al. 1992). This confirms the dust hypothesis for this source, which Clements et al. claim to be an extreme starburst (rather than a primeval galaxy or dust-enshrounded quasar).

The far-infrared luminosity of $\mathrm{H} 1413+117$ is quite large. Taking $L_{\mathrm{FIR}}=v_{\mathrm{em}} L_{v_{\mathrm{em}}}\left(v_{\mathrm{em}}=\right.$ emitted frequency) for the 345 $\mu \mathrm{m}$ point, we find $L_{\mathrm{FIR}} \sim 1 \times 10^{13} L_{\odot}\left(H_{0}=75 \mathrm{~km} \mathrm{~s}{ }^{-1}\right.$ $\mathrm{Mpc}^{-1}, q_{0}=0.5$ ). If the submillimeter flux of $\mathrm{H} 1413+117$ is emitted by dust, we can calculate a dust mass using $M_{\text {dust }}=$ $\left(L_{\mathrm{FIR}} / 10^{4} L_{\odot}\right)\left(40 \mathrm{~K} / T_{\text {dust }}\right)^{5} M_{\odot}$ (Sanders, Scoville, \& Soifer 1991). Assuming $T_{\text {dust }}=40 \mathrm{~K}$, we find $M_{\text {dust }} \sim 1 \times 10^{9} M_{\odot}$. This is lower than the dust mass $\left(9 \times 10^{9} M_{\odot}\right.$ for $H_{0}=75 \mathrm{~km}$ $\mathrm{s}^{-1} \mathrm{Mpc}^{-1}$ ) calculated for IR $10214+4724$ by Clements et al. (1992), based on the rest infrared spectrum between about 25 and $250 \mu \mathrm{m}$.

\section{SUMMARY AND CONCLUSIONS}

In this Letter we have reported submillimeter continuum observations of six radio-quiet $I R A S \mathrm{AGNs}$, with the aim of testing models for the infrared continuum by constraining the far-infrared/submillimeter cutoff slope. The measured cutoff slopes in three of these objects are $\geq 2.50$, thereby ruling out self-absorbed synchrotron emission from electrons with a single power-law energy distribution. Synchrotron emission from sources with more complex electron distributions cannot yet be rejected, but such sources must also be completely homogeneous in order to satisfy the slope constraints set by us and by others. We conclude that the cutoff slope results are consistent with, and favor, the dust model for the infrared continuum.

A related program of measurements of broad-absorptionline quasars has also yielded an interesting cutoff slope result, which we have reported here as well. This result is for the $z=2.546$ gravitationally lensed BAL quasar $\mathrm{H} 1413+117$, for which we measured a slope of 2.95 between 438 and $761 \mu \mathrm{m}$ (124 and $215 \mu \mathrm{m}$ rest frame). If this cutoff is similar in origin to those seen in nearby $I R A S$ quasars, and not caused by absorption in an intervening gas, then we may be seeing a very luminous but otherwise rather normal appearing far-infrared continuum in this very distant object. There are a number of other high-redshift ( $z \gtrsim 2.5$ ) quasars known with optical magnitudes as bright or brighter than $\mathrm{H} 1413+117$, for which the $100 \mu \mathrm{m}$ far-infrared peak might also be detectable in the submillimeter region. We plan to pursue further observations of $\mathrm{H} 1413+117$ and other bright, high-redshift objects, in order to learn more about the far-infrared continua and cutoff slopes in highly luminous radio-quiet quasars.

The authors would like to acknowledge generous allocations of observing time for this project by the JCMT TAC, and assistance from the JCMT support scientists and telescope operators, especially Bill Duncan. We also thank the referee, Rick Edelson, for useful suggestions. R. A. acknowledges partial support from the NSF grant NSFAS T91-20053.
Barvainis, R. 1987, ApJ, 350, 537 1990, ApJ, 353, 419

1992, in Testing the AGN Paradigm, ed. S. Holt, S. Neff, \& M. Urry (New York: AIP) 129

Baryainis, R., \& Antonucci, R. 1989, ApJS, 70, 257

Chini, R., Kreysa, E., \& Beirmann, P. L. 1989, A\&A, 219, 87

Clements, D. L., Rowan-Robinson, M., Lawrence, A., Broadhurst, T., \& McMahon, R. 1992, MNRAS, 256, 35P

Coleman, P. 1992, in preparation

de Kool, M., \& Begelman, M. C. 1989, ApJ, 345, 135

Draine, B. T., \& Lee, H. M. 1984, ApJ, 285, 89

Duncan, W. D., Robson, E. I., Ade, P. A. R., Griffin, M. J., \& Sandell, G. 1990, MNRAS, 243,126

Edelson, R. A., Gear, W. K. P., Malkan, M. A., \& Robson, E. I. 1988, Nature, 336,749

\section{REFERENCES}

Edelson, R. A., \& Malkan, M. A. 1986, ApJ, 308, 59

Low, F. J., Huchra, J. P., Kleinmann, S. G., \& Cutri, R. M. 1988, ApJ, 327, L41 Magain, P., Surdev, J., Swings, J.-P., Borgeest, U., Kayser, R., Kuhr, H., Refsdal, S., \& Remy, M. 1988, Nature, 334, 325

Marscher, A. P. 1979, ApJ, 216, 224

Neugebauer, G., Miley, G. K., Soifer, B. T., \& Clegg, P. E. 1986, ApJ, 308, 815

Rudy, R. J., LeVan, P. D., \& Rodriguez-Espinopsa, J. M. 1982, AJ, 87, 598

Sanders, D. B., Phinney, E. S., Neugebauer, G., Soifer, B. T., \& Matthews, K. 1989, ApJ, 347, 29

Sanders, D. B., Scoville, N. Z., \& Soifer, B. T. 1991, ApJ, 370, 158

Schlickeiser, R., Biermann, P. L., \& Crusius-Watzel, A. 1991, A\&A, 247, 283

Schmidt, M., \& Green, R. F. 1983, ApJ, 269, 352

Veron-Cetty, M.-P., \& Veron, P. 1989, ESO Scientific Report No. 7, A Catalogue of Quasars and Active Nuclei, 4th ed. 